\title{
Monitoring of gentamicin blood level in one-week-of-life neonates admitted to a special care nursery ward
}

\author{
(1) Suzana Mustafa ${ }^{1}$, (1) Siti Latifah Azirah Mohd Noor ${ }^{1}$, (1) Rozitah Razman², (1) Wan Nazirah Wan Yusuf ${ }^{3}$ \\ ${ }^{1}$ Hospital Raja Perempuan Zainab II, Ministry of Health, Department of Pharmacy, Kota Bharu, Malaysia \\ ${ }^{2}$ Hospital Raja Perempuan Zainab II, Ministry of Health, Department of Paediatrics, Kota Bharu, Malaysia \\ 3Universiti Sains Malaysia, Department of Pharmacology, Kelantan, Malaysia
}

\section{Date submitted:}

13.12.2020

Date accepted:

16.02.2021

Online publication date:

15.03.2022

\section{Corresponding Author:}

Suzana Mustafa, PhD, Hospital Raja

Perempuan Zainab II, Ministry of

Health, Department of Pharmacy, Kota

Bharu, Malaysia

szn_m@yahoo.com

ORCID:

orcid.org/0000-0002-5025-2308

Keywords: Gentamicin, neonates, therapeutic drug monitoring

\begin{abstract}
Aims: Gentamicin is routinely used in neonates as an empirical antibiotic for suspected sepsis at a dose of $4.0 \mathrm{mg} / \mathrm{kg}$, either 24 or 36 hourly based on premenstrual age. Regularly, therapeutic drug monitoring is performed. This study was conducted to determine gentamicin blood level in one-week-of-life neonates on $4 \mathrm{mg} / \mathrm{kg}$ treatment.
\end{abstract}

Methods: Neonates who received gentamicin in a special care nursery ward were identified from the records retrospectively. The included subjects were neonates between 3 and 7 days old treated with gentamicin for at least three days. Admission diagnoses, dosing protocol (including dosage, the timing of the doses, and timing of blood samples) and measured gentamicin trough and peak levels were recorded.

Results: A total of 290 neonates met the inclusion criteria (male: $57.6 \%$ ). Over $30 \%$ of the subjects treated with gentamicin experienced potential toxicity with trough levels above $1.0 \mu \mathrm{g} / \mathrm{mL}$, and $15.9 \%$ of them had sub efficacy of the drug (peak level below $5 \mu \mathrm{g} / \mathrm{mL}$ ). Six neonates had trough levels above $2.0 \mu \mathrm{g} / \mathrm{mL}$. The percentage of potential toxicity was higher in subjects with presumed sepsis, in those with bodyweight between $2.5-2.99 \mathrm{~kg}$ and with 24hour dosing interval.

Conclusions: We propose a trough-only monitoring protocol in non-critically ill neonates, as the practice of monitoring pre and post often necessitate additional blood sampling. The decision of not determining the peak levels routinely can be based on the outcome that gentamicin dosage of $4.0 \mathrm{mg} / \mathrm{kg}$ likely provides peak levels in desired range without any added risk of toxicity.

\section{Introduction}

Gentamicin is routinely used in neonates as an empirical antibiotic for suspected sepsis. It is given together with ampicillin or penicillin (cloxacillin if a staphylococcal infection is suspected) as recommended by World Health Organization. Due to its narrow therapeutic index, gentamicin required therapeutic drug monitoring (TDM) during treatment to ensure efficacy, reduce the potential for ototoxicity and nephrotoxicity, and reduce the potential for resistance.

There is considerable variation in dosing regimen guidelines, but the common dosage regimen used is between 4 and $7 \mathrm{mg} /$ kg every 24 to $36 \mathrm{~h}$, depending on the gestational age. In a study from Thailand, once-daily gentamicin therapy of $4 \mathrm{mg} / \mathrm{kg}$ in neonates of more than 34-week gestation resulted in appropriate peak and lower trough concentrations (1). The study suggested that routine collection of serum gentamicin concentration may not be necessary for neonates with normal renal function receiving a 3-day, once-daily gentamicin of $4 \mathrm{mg} / \mathrm{kg}$ for suspected sepsis. It was concluded that once-daily gentamicin dosing was preferable to any other dosing interval because of the simplicity of the regimen, which achieves desirable drug levels (1). In another study, neonates who received once-daily gentamicin at a dose of $5 \mathrm{mg} / \mathrm{kg} /$ day reported unacceptably high trough levels (2). 
In Raja Perempuan Zainab II Hospital (HRPZ II), stable neonates with risk factors associated with neonatal sepsis such as maternal Group B Streptococcus infection, maternal fever, low birth weight, or preterm are treated in Special Care Nursery (SCN) Ward. Gentamicin is prescribed empirically combined with a penicillin drug, at a dose of $4.0 \mathrm{mg} / \mathrm{kg}$, either 24 or 36 hourly based on the premenstrual age. Regularly, TDM is used for both trough and peak samples of gentamicin in SCN. In this study, we examined the peak and trough serum gentamicin level in one-week-of-life neonates on a $4 \mathrm{mg} / \mathrm{kg}$ dosing schedule.

\section{Methods}

In this single-center and retrospective study, neonates who received gentamicin at the SCN ward were identified from the database of TDM 2019 records for a one-year interval. TDM forms were reviewed for diagnoses, dosing history, including dosage, the timing of the doses, and timing of blood samples, and measured gentamicin trough and peak levels (Figure 1). We only included neonates between 3 and 7 days old admitted to the SCN ward in 2019 who were treated with gentamicin for at least three days. The study was approved by the Medical Research and Ethics Committee of the Ministry of Health, Malaysia (NMRR-20-254-53622 IIR date: 09.04.2020). Informed consent was not required due to the retrospective design of the study. Patients' information was anonymized and de-identified before analyses. The study protocol conforms to the 1975 Helsinki Declaration.

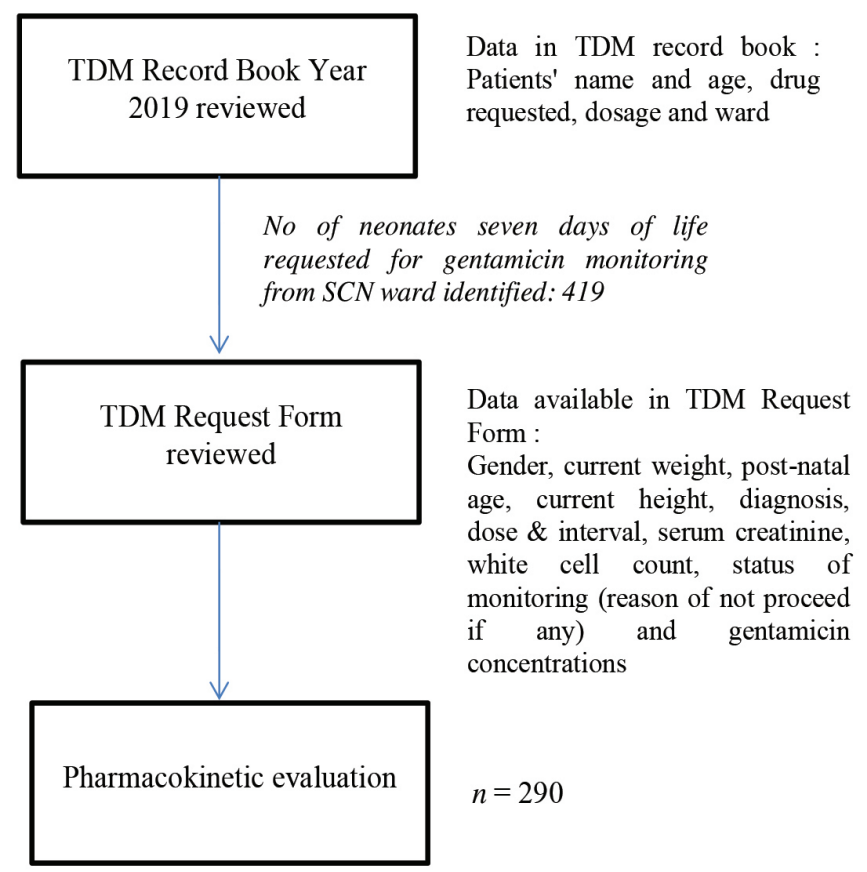

Figure 1. Flow chart of patients recruitment

TDM: Therapeutic drug monitoring
Neonates treated with gentamicin received an initial dose of $3.5-4 \mathrm{mg} / \mathrm{kg}$ daily. Gentamicin was diluted and infused over 60 min according to the calculated doses. TDM was performed by measuring the peak and trough gentamicin blood level $1.0 \mathrm{~h}$ and 23.5h or $35.5 \mathrm{~h}$ after the infusion of the third dose.

The trough serum gentamicin level was measured 30 min before the third dose, and the peak was measured 60 min after infusion on the third dose. Determination of gentamicin was performed using the COBAS INTEGRA analyzer according to the instructions provided in the manufacturer's manual. The steady-state Sawchuk-Zaske method was used to calculate the actual trough and peak levels (3). Peak is associated with efficacy of gentamicin while trough is associated with gentamicin toxicity.

\section{Statistical Analysis}

Descriptive statistics were performed using Statistical Package for the Social Sciences version 22.0 (Armonk, NY). Data are expressed as the mean \pm standard deviation and median. The chi-square test was used to analyze categorical data, and simple linear regression was carried out to investigate the relationship between post-natal age, weight, and creatinine clearance with gentamicin concentrations. $P<0.05$ indicated statistical significance.

\section{Results}

\section{Patients}

Overall, 419 TDM requests were reviewed and 291 cases were analyzed. One hundred twenty-eight cases were not included in the analyses because blood specimens exceeded stability time $(n=89)$, and insufficient samples were obtained $(n=39)$. Another admission was also excluded due to missing documentation of age. Table 1 shows the demographic data. Presumed sepsis, clinical sepsis, and congenital pneumonia were the common clinical diagnoses (Table 2).

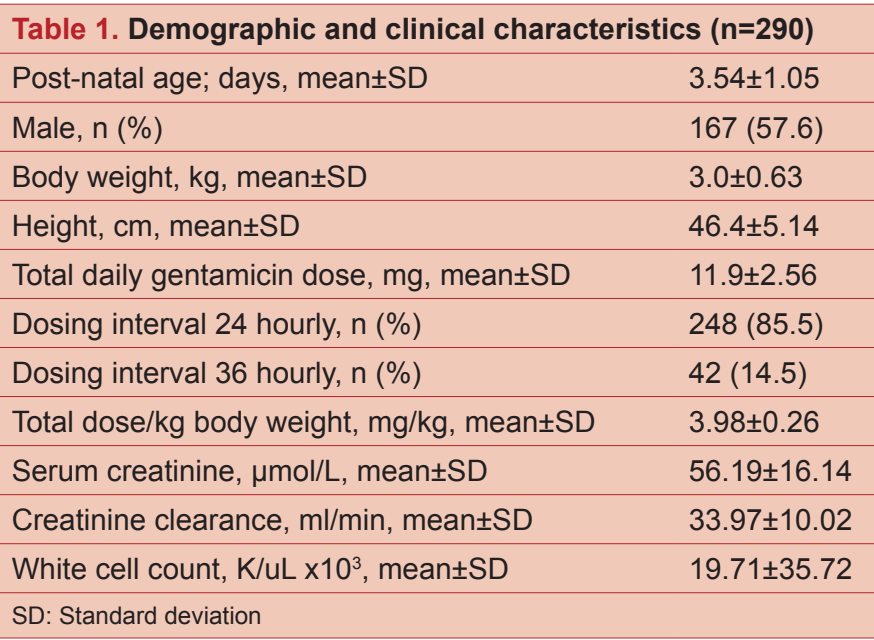




\section{Gentamicin serum concentrations}

Gentamicin was started in the majority of the neonates on the first days of life. Table 3 shows gentamicin serum concentrations. A trough level above $1.0 \mu \mathrm{g} / \mathrm{mL}$ is considered potentially toxic, and a peak level of $5.0 \mu \mathrm{g} / \mathrm{mL}$ and above is considered effective. Renal function (presented as creatinine clearance) showed a negative relationship with the trough level of gentamicin. The same relationship was also observed between the weight of neonates and the peak level of gentamicin (Table 3).

\begin{tabular}{|c|c|}
\hline \multicolumn{2}{|c|}{$\begin{array}{l}\text { Table 2. Initial diagnosis of neonates before starting empirical } \\
\text { gentamicin }(n=290)\end{array}$} \\
\hline Presumed sepsis, n (\%) & $108(37.2)$ \\
\hline Clinical sepsis, n (\%) & $79(27.2)$ \\
\hline Congenital pneumonia, $\mathrm{n}(\%)$ & $73(25.2)$ \\
\hline Respiratory distress syndrome, $\mathrm{n}(\%)$ & $23(7.9)$ \\
\hline Group B Streptococcus pneumoniae, n (\%) & $5(1.7)$ \\
\hline Community acquired pneumonia, $\mathrm{n}(\%)$ & $2(0.7)$ \\
\hline
\end{tabular}

Concomitant antibiotics were documented in 165 cases. Most of the combined antibiotic therapy included ampicillin or penicillin.

Over $30 \%$ of neonates treated with gentamicin experienced potential toxicity with trough levels above $1.0 \mu \mathrm{g} / \mathrm{mL}$, and $15.9 \%$ of them had sub-efficacy of the drug (peak level below $5 \mu \mathrm{g} /$ $\mathrm{mL}$ ). Only six neonates had trough levels above $2.0 \mu \mathrm{g} / \mathrm{mL}$. The percentage of potential toxicity was higher in presumed sepsis neonates; in subjects with bodyweight between $2.5-2.99 \mathrm{~kg}$ and in subjects who received 24-hour dosing (Table 4).

\section{Discussion}

Gentamicin is one of the most commonly prescribed antibiotics for culture-proven or suspected sepsis in neonates. Since the development and organ maturation influences gentamicin pharmacokinetics in neonates, interest has increased in refining dosing regimens for improved efficacy and decreased toxicity. In this population, variability in kidney function and body composition, in particular, is responsible for the large interpatient variability in clearance and volume of distribution of gentamicin.

\begin{tabular}{|c|c|c|c|c|}
\hline & \multicolumn{2}{|c|}{ Gentamicin trough concentration ${ }^{a}$} & \multicolumn{2}{|c|}{ Gentamicin peak concentration ${ }^{a}$} \\
\hline & Beta $(95 \% \mathrm{Cl})$ & $\mathbf{p}$ & Beta $(95 \% \mathrm{Cl})$ & $\mathbf{p}$ \\
\hline Weight & $-0.035(-0.157,0.087)$ & 0.575 & $-0.025(-0.045,-0.006)$ & 0.012 \\
\hline Creatinine clearance & $-3.66(-6.79,-0.534)$ & 0.022 & $-0.562(-1.18,0.057)$ & 0.075 \\
\hline
\end{tabular}

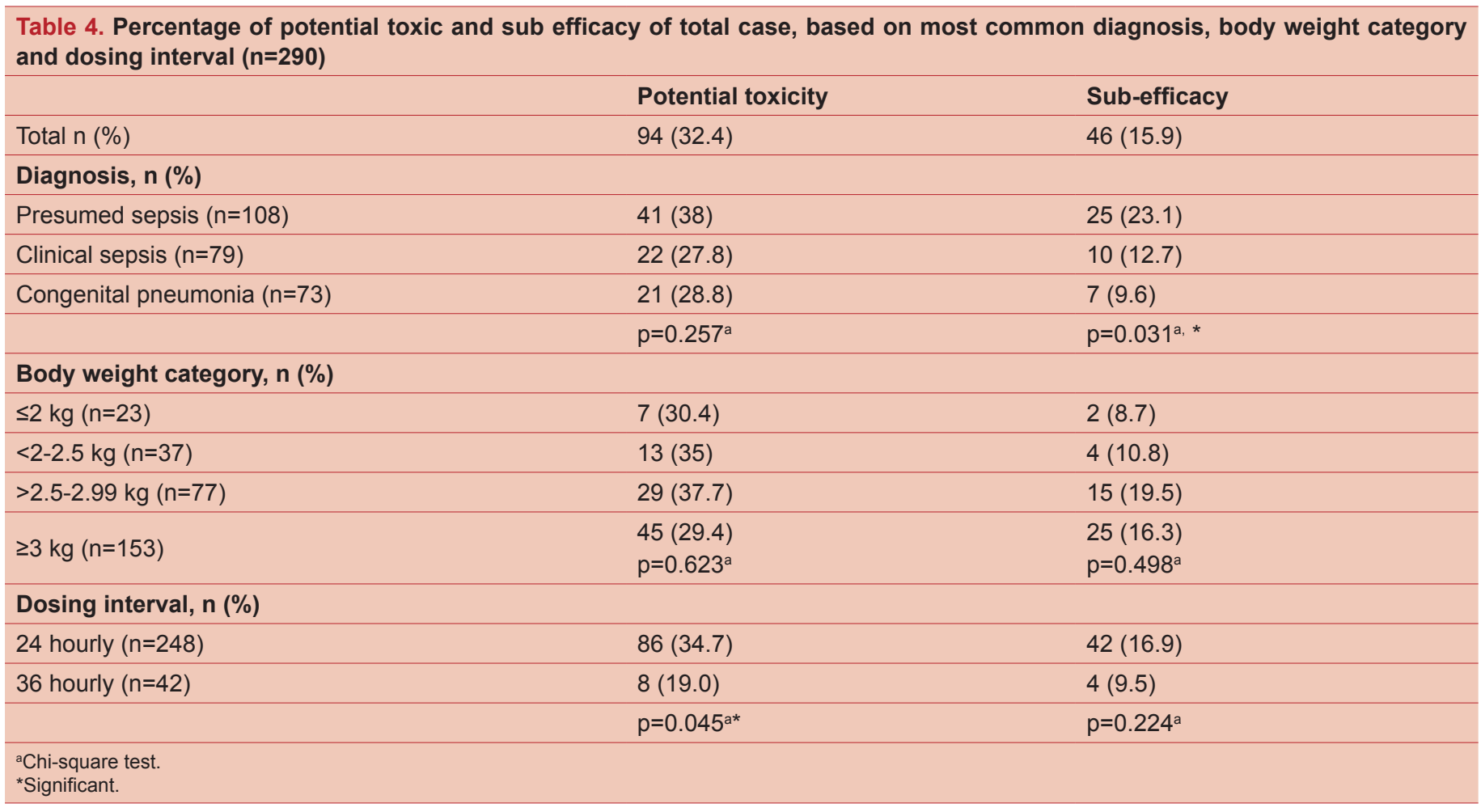


The clearance of gentamicin is almost entirely dependent on glomerular filtration (4).

In this retrospective study, the rejection rate of gentamicin analysis in SCN neonates over one year was $30.5 \%$. Most of the samples $(69.5 \%)$ were rejected due to delays in the transport of the sample to the laboratory, which may cause erroneous test results. Inaccurate gentamicin levels may cause missinterpretation and consequently inappropriate management as either under-treatment or over-treatment that leads to nephrotoxicity or ototoxicity. Therefore, delayed samples were not analyzed.

Delayed transport of the sample is a human error but the percentage was quite high in the current study. The blood samples (especially pre-level) were kept in the wards for more than four hours before transport to the laboratory. The reason for the delay in the transportation of blood samples was waiting for the post-level samples from the same patient. Delayed administration of gentamicin or difficulty in taking blood samples were also among the probable causes. Other than delays in transportation, insufficient blood samples (30.5\%) was another reason for the rejection of the sample by the laboratory. Insufficient means that there was not enough specimen volume to perform the TDM assay.

Specimen rejection and subsequent recollection require extra communication between the pharmacist, medical laboratory technician, and the clinician, extra working hours, and additional resources for recollection and reanalysis, resulting in discomfort and stress imposed on neonates. The blood volume of a neonate is approximately 75 to $107 \mathrm{~mL} / \mathrm{kg}$ (5). Small neonates may have a total blood volume as low as $50 \mathrm{~mL}$. This means drawing just 1.0-mL blood from a neonate could significantly reduce his or her blood volume. Blood loss attributable to laboratory testing is acknowledged as the primary factor leading to anemia in infants in the first weeks of life (6). Therefore, it is important to reduce the number of unnecessary gentamicin blood sampling.

Optimal dosing of gentamicin in neonates is still a matter of debate although it is commonly used. In our hospital setting, $4.0 \mathrm{mg} / \mathrm{kg}$ of gentamicin is given as once-daily dosing for fullterm neonates and 36 hourly for pre-term neonates according to local guidelines (7). With this protocol, $84.1 \%$ achieve an appropriate peak of at least $5.0 \mu \mathrm{g} / \mathrm{mL}$. Thai neonates also achieved an appropriate peak with a similar dosage whereby $97 \%$ of their neonates had a peak above $4.0 \mu \mathrm{g} / \mathrm{mL}$ (1). The percentage increased to $100 \%$ when gentamicin dosage was increased to $5.0 \mathrm{mg} / \mathrm{kg}$ but $21 \%$ of the Thai neonates had higher peak levels above $12.0 \mu \mathrm{g} / \mathrm{mL}$ (2). The generally accepted peak concentrations are $4.0-10.0 \mu \mathrm{g} / \mathrm{mL}$ in neonates, however, many guidelines and researchers target peaks above $5.0 \mu \mathrm{g} / \mathrm{mL}$ for optimization of gentamicin effectiveness (8).

In our setting, $92.8 \%$ of the neonates had a peak concentration above $4.0 \mu \mathrm{g} / \mathrm{mL}$. Collectively, these findings suggest that the current practice of $4.0 \mathrm{mg} / \mathrm{kg}$ dosage is appropriate to achieve the desired peak level in our population. A pharmacokineticpharmacodynamic model that describes the time course of the bactericidal activity of gentamicin and its adaptive resistance in neonates also demonstrated that $4.0 \mathrm{mg} / \mathrm{kg}$ of gentamicin every 24 hours is the most effective in the newborn infant as the bacteria remained below the starting inoculum for the longest time. The model also supported an extended dosing interval of 36 to 48 hourly in preterm neonates that were as effective as a 24 hourly dosing interval for the same total dose (9). In fullterm neonates, the bacterial count reached the initial starting inoculum $(5 \times 105 \mathrm{CFU} / \mathrm{mL})$ in 48 hours for $4.0 \mathrm{mg} / \mathrm{kg}$ every 24 hours. The model also predicted using different initial doses of 4.0 to $7.0 \mathrm{mg} / \mathrm{kg}$ for the extreme preterm (gestational age of 25 weeks) and term (gestational age of 40 weeks) neonates and displayed minor differences in bacteria-killing between the different doses during the first $6 \mathrm{~h}$ (9).

In our setting, trough level is targeted at below $1.0 \mu \mathrm{g} / \mathrm{mL}$ since trough level of more than $1.0 \mathrm{mg} / \mathrm{L}$ suggests accumulation (potentially toxic) and above $2.0 \mu \mathrm{g} / \mathrm{mL}$ is toxic (10). Based on the target, the percentage of a potential toxic incident in overall neonates with a dosage of $4.0 \mathrm{mg} / \mathrm{kg}$ is $32.4 \%$ and is between $19-38 \%$ according to the most common diagnoses, body weight, or dosing interval. Gestational age was not documented in TDM forms reviewed, therefore we were uncertain whether neonates with a bodyweight of two kilograms and below were premature or not. All three neonates with 24 hourly intervals had potential toxic trough levels (above $1.0 \mu \mathrm{g} / \mathrm{mL}$ ). One neonate with a bodyweight of more than 3 kilograms was prescribed with 36 hourly intervals and his trough level was not detected. This is indicative that 36 hourly interval is inappropriate for the body weight. Our findings are suggestive of 36 hourly intervals for neonates of $2.0 \mathrm{~kg}$ and below regardless of gestational age. Increasing the interval between doses can maintain maximal antibacterial activity while minimizing side effects.

The percentage of neonates with trough levels above 2.0 $\mu \mathrm{g} / \mathrm{mL}$ was comparably low in our population compared to Thai neonates whereby incidence was only $2.0 \%$. In Thai neonates, the incidence is $6.67 \%$ (1). The principal adverse effects of gentamicin therapy are renal toxicity, which nearly always is reversible, and ototoxicity which generally is irreversible. The amount of drug that accumulates in the kidney and inner ear increases with higher plasma concentrations and longer periods of exposure. The elimination of a drug from these organs, on the other hand, occurs more slowly than from plasma and is retarded by high plasma concentrations. Back diffusion from these sensitive organs to the blood, thereby lowering end-organ drug levels, is dependent primarily on the trough rather than the peak concentration of the drug in the serum. This accounts for the association between toxicity and high plasma trough concentrations. Once-daily dosing or extended-interval dosing, 
despite the higher peak concentration, provides a longer period when serum concentrations are below the threshold for toxicity, leading to a reduced risk of toxicity (10).

Current findings suggest that the monitorization of trough levels alone is generally sufficient in non-critically ill neonates treated in the SCN to verify that gentamicin is being adequately eliminated. Peak levels are typically not required, as the larger doses used are expected to produce concentrations well above those required for clinical efficacy.

Limitations of the current study include the small volume of drug administered to neonates less than $2.0 \mathrm{~kg}$, with possible administration error, which influences the reported concentrations, and impacts on subsequent pharmacokinetic calculations. Other limitations relate to clinical acuities such as hydration levels, gestational age, and birth weight which were not documented in the TDM request form. Patients' medical records were not reviewed due to time constraints, therefore data on the culture of infection was not available to confirm infection.

\section{Conclusion}

We propose trough-only monitoring in non-critically ill neonates admitted to SCN as the current practice of monitoring pre and post often necessitates additional blood sampling. The decision not to determine peak levels routinely is based on the present outcome that gentamicin dosage of $4.0 \mathrm{mg} / \mathrm{kg}$ is likely to provide peak levels in desired range without any added risk of toxicity, lesser trauma, and blood sampling.

\section{Acknowledgments}

We would like to thank the Director General of Health Malaysia for his permission to publish this article. Sincere appreciation also goes to the director of Hospital Raja Perempuan Zainab II for her assistance and support.

\section{Ethics}

Ethics Committee Approval: This research was conducted in accordance with the 1975 Helsinki Declaration and approval from Medical Research and Ethics Committee of Ministry of Health, Malaysia (NMRR-20-254-53622 IIR, date: 09.04.2020).

Informed Consent: Retrospective study.

Peer-review: Externally peer-reviewed.

\section{Authorship Contributions}

Surgical and Medical Practices: R.R., Concept: S.M., R.R., W.N.W.Y., Design: S.M., R.R., W.N.W.Y., Data Collection or Processing: S.M., S.L.A.M.N., Analysis or Interpretation: S.M., S.L.A.M.N., W.N.W.Y., Literature Search: S.M., S.L.A.M.N., Writing: S.M., S.L.A.M.N., R.R., W.N.W.Y.

Conflict of Interest: No conflict of interest was declared by the authors.

Financial Disclosure: The authors declared that this study received no financial support.

\section{References}

1. Kiatchoosakun $P$, Kosalaraksa P, Jirapradittha J, Taksaphan S, Tassniyom S. Once-Daily Gentamicin Dosing of $4 \mathrm{Mg} / \mathrm{Kg} /$ Dose in Neonates. J Med Assoc Thai. 2017;88:934-938.

2. Kosalaraksa $P$, Janthep $P$, Jirapradittha J, Taksaphan S, Kiatchoosakun P. Once versus twice daily dose of gentamicin therapy in Thai neonates. J Med Assoc Thai. 2004;87:372-376.

3. Lackner TE, Lyss AP. A simple method to estimate the initial dose of gentamicin. Pharmacotherapy. 1992;12:195-200.

4. van Donge T, Pfister M, Bielicki J, et al. Quantitative Analysis of Gentamicin Exposure in Neonates and infants calls into question its current dosing recommendations. Antimicrob Agents Chemother. 2018;62:e02004.

5. Usher R, Shephard M, Lind J. The Blood Volume of the Newborn Infant and Placental Transfusion. Acta Paediatr. 1963;52:497-512.

6. Lin JC, Strauss RG, Kulhavy JC, et al. Phlebotomy overdraw in the neonatal intensive care nursery. Pediatrics. 2000;106:19.

7. Clinical Pharmacokinetics Pharmacy Handbook. 2nd ed. Pharmacy Practice \& Development Division, $\mathrm{MOH}$ Malaysia; 2019. https://www.pharmacy.gov.my/v2/sites/default/files/ document-upload/clinical-pharmacokinetics-pharmacyhandbook-ccph-2nd-edition-rev-2.0.pdf

8. Valitalo PA, van den Anker JN, Allegaert K, et al. Novel modelbased dosing guidelines for gentamicin and tobramycin in preterm and term neonates. J Antimicrob Chemother. 2015:70:2074-2077

9. Mohamed AF, Nielsen El, Cars O, Friberg LE. Pharmacokineticpharmacodynamic model for gentamicin and its adaptive resistance with predictions of dosing schedules in newborn infants. Antimicrob Agents Chemother. 2012;56:179-188.

10. Darmstadt GL, Miller-Bell M, Batra M, Law P, Law K. Extendedinterval dosing of gentamicin for treatment of neonatal sepsis in developed and developing countries. J Health Popul Nutr. 2008;26:163-182. 